\title{
Association between Plasma Homocysteine Concentrations and the First Ischemic Stroke in Hypertensive Patients with Obstructive Sleep Apnea: A 7-Year Retrospective Cohort Study from China
}

\author{
Nanfang Li $\mathbb{D}^{1},{ }^{1}$ Xintian Cai ${ }^{(i)},{ }^{2}$ Qing Zhu, ${ }^{2}$ Xiaoguang Yao, ${ }^{1}$ Mengyue Lin, ${ }^{2}$ Lin Gan, \\ Le Sun, ${ }^{1}$ Na Yue, ${ }^{1}$ Yingli Ren, ${ }^{1}$ Jing Hong, ${ }^{1}$ Yue Ma, ${ }^{1}$ Run Wang, ${ }^{1}$ Jina Yili, ${ }^{1}$ and Qin Luo ${ }^{1}$ \\ ${ }^{1}$ Hypertension Center of People's Hospital of Xinjiang Uygur Autonomous Region, Xinjiang Hypertension Institute, National Health \\ Committee Key Laboratory of Hypertension Clinical Research, Xinjiang Clinical Medical Research Center for \\ Hypertension Diseases, Urumqi, Xinjiang, China \\ ${ }^{2}$ Xinjiang Medical University, Urumqi, Xinjiang, China
}

Correspondence should be addressed to Nanfang Li; lnanfang2016@sina.com and Xintian Cai; 278996834@qq.com

Received 24 August 2021; Revised 16 September 2021; Accepted 21 September 2021; Published 28 September 2021

Academic Editor: Wen-Jun Tu

Copyright (c) 2021 Nanfang Li et al. This is an open access article distributed under the Creative Commons Attribution License, which permits unrestricted use, distribution, and reproduction in any medium, provided the original work is properly cited.

\begin{abstract}
Purpose. This study was aimed at investigating the association between baseline plasma homocysteine (Hcy) concentrations and the risk of the first ischemic stroke (IS) and at investigating any possible influential modifying factors in hypertensive patients with obstructive sleep apnea (OSA). Methods. Cox proportional hazards regression was employed to investigate the relationship between plasma Hcy concentration and the first IS. A generalized additive model was applied to determine the nonlinear relationship. In addition, we conducted subgroup analysis. Results. A total of 2350 hypertensive patients with OSA without a history of IS were enrolled in this study. At a median follow-up of 7.15 years, we identified 93 cases of the first IS. After adjusting for potential confounding, the findings revealed that plasma Hcy concentration was strongly and positively associated with the occurrence of the first IS (per SD increment; HR $=1.37,95 \% \mathrm{CI}$ : 1.30-1.44). A nonlinear relationship was found between plasma Hcy concentration and the risk of developing the first IS with inflection points for plasma Hcy of $5 \mu \mathrm{mol} / \mathrm{L}$. In stratified analysis, a greater positive correlation was found between baseline plasma Hcy concentrations and new-onset IS in patients with $\mathrm{DBP} \geq 90 \mathrm{mmHg}$ (per SD increment; $\mathrm{HR}=1.48,95 \% \mathrm{CI}: 1.33-1.65$ vs. $<90 \mathrm{mmHg}$ : $\mathrm{HR}=1.20,95 \% \mathrm{CI}: 1.02-1.42$; $P$-interaction $=0.04$ ) and BMI $\geq 24$ and $<28 \mathrm{~kg} / \mathrm{m}^{2}$ (per SD increment; $\mathrm{HR}=1.46,95 \% \mathrm{CI}: 1.26-1.70 \mathrm{vs} .<24 \mathrm{~kg} / \mathrm{m}^{2}: \mathrm{HR}=1.13$, 95\% CI: $0.95-1.33$ vs. $\geq 28 \mathrm{~kg} / \mathrm{m}^{2}: \mathrm{HR}=1.46,95 \%$ CI: $1.25-1.70 ; P$-interaction $\left.=0.03\right)$. Conclusion. Elevated plasma Hcy concentrations are independently associated with the risk of the first IS in hypertensive patients with OSA. Plasma Hcy concentrations $\geq 5 \mu \mathrm{mol} / \mathrm{L}$ surely increased the risk of the first IS in hypertensive patients with OSA.
\end{abstract}

\section{Introduction}

Stroke is the leading cause of disability worldwide and the second leading cause of death after ischemic heart disease [1-3]. More importantly, according to China's National Disease Surveillance Point System, there were an estimated 2.4 million new-onset strokes and 1.1 million stroke-related deaths per year [4]. Researches have demonstrated that ischemic stroke (IS) is the most prevalent stroke subtype in
China, accounting for about $70-86 \%$ of all strokes $[2,5]$. Therefore, early identification and management of associated risk factors are critical measures to prevent and treat the first IS $[6,7]$.

Obstructive sleep apnea (OSA) is a widespread sleep disturbance $[8,9]$. The high prevalence of OSA in hypertensive patients is well demonstrated, and OSA is especially prevalent in patients with intractable hypertension [10-12]. Hypertension and OSA often cooccur, which may contribute 
to a significantly enhanced risk of cardiovascular disease in hypertensive patients with OSA [13]. OSA is associated independently with carotid intima-media thickness in hypertensive patients [14]. OSA was additionally associated with an increased risk of new-onset ventricular fibrillation, and a significant dose-response correlation was identified between the severity of OSA and the degree of risk of developing ventricular fibrillation [15]. A meta-analysis of retrospective researches revealed that hypertensive patients with comorbid moderate to severe OSA had significantly increased mortality from cardiovascular disease compared with those without comorbid OSA $[16,17]$. A few reports have indicated that people with hypertension combined with OSA have a two-fold elevated risk of developing IS compared to people without OSA [16-18].

Although previous investigations have reported a significant association between hyperhomocysteinemia (HHcy) caused by disorders of homocysteine (Hcy) metabolism and the first IS, the relationship between the degree of Hcy metabolism disorder and the first IS remains controversial [19]. In general, the normal reference range for plasma Hcy concentrations is 5 to $10 \mu \mathrm{mol} / \mathrm{L}$. Thus, the definition of HHcy is disputable and is commonly defined as plasma Hcy concentrations $\geq 10 \mu \mathrm{mol} / \mathrm{L}$, but also as $\mathrm{Hcy} \geq 15 \mu \mathrm{mol}$ /L [20-23]. Current guidelines suggest plasma Hcy concentration control goals only in people with pure hypertension to prevent IS events [24]. However, available data from cohort investigations on the correlation between baseline plasma Hcy concentrations and incident first IS are limited and inconclusive, especially in hypertensive patients with OSA. It is worth noting that previous investigations have rarely thoroughly examined the potential moderators of the relationship between baseline plasma Hcy concentrations and incident first IS risk.

Therefore, we intended to evaluate the association between baseline plasma Hcy concentrations and the first IS and to investigate the appropriate plasma Hcy concentration target to reduce the incidence of the first IS in hypertensive patients with OSA.

\section{Materials and Methods}

2.1. Study Design. This retrospective cohort study included consecutive hypertensive patients with suspected OSA hospitalized at the Hypertension Center of People's Hospital of Xinjiang Uygur Autonomous Region from January 2011 to December 2013. The study protocol was authorized by the Medical Ethics Committee of the People's Hospital of Xinjiang Uygur Autonomous Region (no. 2019030662) and was conducted in adherence to the approved guidelines. Because of the retrospective nature of the study, patient consent and/or informed consent was not required.

A total of 2585 patients aged $\geq 18$ years with hypertension combined with OSA determined by polysomnography (PSG) were enrolled in this study. Exclusion criteria for this study were age younger than 18 years $(n=11)$, severe systemic disease $(n=25)$ (i.e., severe pulmonary disease, malignancy, severe liver disease, or severe chronic kidney disease or other major diseases that affect long-term survival), cen- tral sleep apnea $(n=12)$, pregnancy $(n=6)$, lacking a fasting blood sample and physical examination at baseline $(n=61)$, a history of stroke at baseline $(n=24)$, and loss to follow-up $(n=96)$. Ultimately, 2350 study participants were eligible for inclusion in the statistical analysis.

2.2. Baseline Examination. All participants finished the baseline examination between January 2011 and December 2013. Data on demographic features, lifestyle, personal disease records, history of regular CPAP treatment, and medication history were collected from all candidates through interviews.

Weight, height, neck circumference (NC), and waist circumference (WC) were measured three times, and the mean of the three times measurements was used for analysis. Systolic blood pressure (SBP) and diastolic blood pressure (DBP) were monitored using an electronic sphygmomanometer (HEM-1000, Omron, Kyoto, Japan). All participants underwent PSG examination throughout the night. All participants were instructed to refrain from coffee, alcohol, and sedative-hypnotics before the sleep study. Interpretation of PSG results was done by a professional polysomnography technician, and all steps were consistent with previous studies $[25,26]$. Venous blood samples were retrieved from the antecubital vein in the early morning after 12 hours of fasting. Fasting plasma glucose (FPG), highdensity lipoprotein (HDL-c), total cholesterol (TC), lowdensity lipoprotein (LDL-c), triglycerides (TG), creatinine (Cr), and high-sensitivity C-reactive protein (hs-CRP) levels were measured. Plasma Hcy concentrations were measured by a fluorescence polarization immunoassay on an automated immunoassay analyzer. The estimated glomerular filtration rate (eGFR) was calculated by using a formula derived from the CKD-EPI [27].

2.3. Definitions. The apnea hypopnea index (AHI) was defined as the sum of apnea and hypopnea events per hour of sleep on average. A diagnosis of OSA was determined as a minimum of 5 events per hour of AHI, with 5 to 14.9 events per hour recognized as mild OSA, 15 to 29.9 events per hour as moderate OSA, and 30 or more events per hour as severe OSA. Hypertension was defined as SBP $\geq 140$ $\mathrm{mmHg}$ and/or $\mathrm{DBP} \geq 90 \mathrm{mmHg}$ and/or previously diagnosed hypertension and/or use of antihypertensive therapy within the past two weeks. Patients with diabetes were defined as those previously diagnosed with diabetes or newly diagnosed with diabetes (fasting glucose $\geq 7.0 \mathrm{mmol} / \mathrm{L}$ on 3 occasions during hospitalization, $\mathrm{HbA} 1 \mathrm{c}>6.5 \%$ at baseline), according to current guidelines. According to the frequency of drinking and smoking, we categorized them as never, former, and current.

2.4. Clinical Outcomes and Follow-Up. Endpoints were acquired through personal interview, records from medical insurance, and hospital discharge summaries. According to former investigations, the principal endpoint was to be determined as the first IS, including cerebral infarction and transient ischemic attack $[28,29]$. The diagnosis of IS was on the basis of a contract vascular computed tomography 


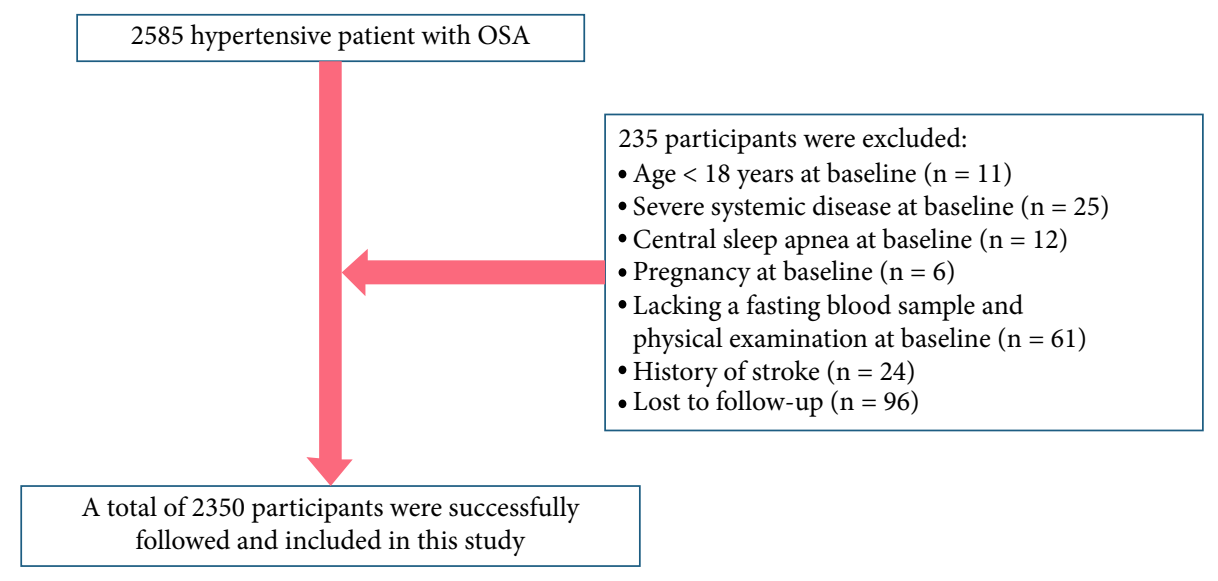

Figure 1: Flowchart.

(CT) scan or cranial CT scan, cerebrovascular angiography, or magnetic resonance imaging of the brain. The period of follow-up started at the first visit and ended on December $31,2020$.

2.5. Statistical Analysis. Differences between baseline characteristics of the different plasma Hcy groups were analyzed by one-way ANOVA, Kruskal-Wallis $H$, and $\chi^{2}$ tests. Multivariate Cox proportional hazard regression was performed to evaluate the association between baseline plasma Hcy concentration and the risk of the first IS by estimating hazard ratios (HR) and 95\% confidence intervals (CI). Adjustment for variables in this study showed varying degrees of adjustment results according to the statement of STROBE [30]. Adjust model I was adjusted for age and gender at baseline; adjusted model II was further adjusted for smoking status, drinking status, NC, BMI, and WC at baseline; adjusted model III was further adjusted for history of arrhythmia, diabetes, coronary heart disease, SBP, DBP, AHI, AI, HI, sleep duration, mean $\mathrm{SaO}_{2}$ and lowest $\mathrm{SaO}_{2}$, antidiabetic drugs, lipid-lowering drugs, antiplatelet drugs, regular CPAP treatment, antihypertensive drugs, and TC, TG, HDL-c, LDL-c, FPG, eGFR, Cr, and hs-CRP levels at baseline. Moreover, we calculated the cumulative IS incidence function of events over time using the Kaplan-Meier method. Further, we simulated the dose-response correlation between plasma $\mathrm{Hcy}$ concentration and IS risk using a generalized additive model, fitted the model with a recursive algorithm using maximum likelihood, and calculated the inflection points for the nonlinear correlation [31]. Considering that association between plasma Hcy concentrations and IS may differ in some populations, we performed exploratory stratified analysis by using a Cox proportional hazards model for some subgroups and used likelihood ratio tests to check for hierarchical differences to determine if there was an interaction. Finally, to estimate the reasonableness of the deviations caused by unmeasured and residual confounding factors, we also calculated the $E$-value of our main research results. The $E$-value estimates the strength of the unmeasured confounding variable needed to invalidate the observed association between our exposure and the result, taking into account all the measured covariables.
All statistical analyses were conducted using $\mathrm{R}$ version 4.0.1 software.

\section{Results}

3.1. Demographic Characteristics. In this cohort study, a total of 2350 participants, including 1611 males and 739 females, were evaluated. Participant screening details are shown in Figure 1. Table 1 shows the baseline characteristics of the participants grouped by plasma Hcy concentration tertile. Overall, the mean age of the 2350 participants was $49.45 \pm$ 10.65 years, and $68.55 \%$ were male.

3.2. Follow-Up Results. During follow-up, 93 (3.96\%) of the hypertensive patients with OSA in the study were diagnosed with IS. Of these, the incidence of IS corresponding to the plasma Hcy concentrations tertile grouping was $1.92 \%$ for T1, 4.99\% for T2, and $4.71 \%$ for T3. Figure 2 illustrates the significant difference in IS risk between plasma Hcy concentration tertile groups (log-rank test, $P=0.0022$ ). The cumulative risk of IS gradually increased with increasing plasma Hcy concentrations.

3.3. Association between Plasma Hcy Concentrations and the First IS in Hypertensive Patients with OSA. The association between plasma Hcy concentrations and the first IS is summarized in Table 2. We found that high plasma Hcy concentrations, expressed as a continuous variable and a categorical variable, were significantly and positively associated with the first IS. When plasma Hcy concentrations were expressed as a continuous variable, plasma Hcy concentrations were significantly associated with first IS in the crude model (per $\mathrm{SD}$ increment; $\mathrm{HR}=1.37,95 \% \mathrm{CI}: 1.30-1.44, P<0.01 ; E-$ value $=2.08)$. In adjust model $\mathrm{I}$, plasma Hcy concentrations were still an independent risk factor for the first IS (per SD increment; $\mathrm{HR}=1.35,95 \% \mathrm{CI}$ : 1.27-1.42, $P<0.01$; E-value $=2.03$ ). In adjust model II, plasma Hcy concentrations were still an independent risk factor for the first IS (per SD increment; $\mathrm{HR}=1.34$, 95\% CI: 1.26-1.43, $P<0.01 ; E$-value $=2.01$ ), as well as the fully adjusted model (adjust model III), where plasma Hcy concentrations (per SD increment; HR $=1.32$, 95\% CI: $1.24-1.38, P<0.01 ; E$-value $=1.97)$ were 
Table 1: Participants sorted by tertiles of Hcy.

\begin{tabular}{|c|c|c|c|c|}
\hline \multirow{2}{*}{ Variable } & \multicolumn{3}{|c|}{ Hcy tertiles $(\mathrm{mmol} / \mathrm{L})$} & \multirow{2}{*}{$P$ value } \\
\hline & Tertile $1(<11.6)$ & Tertile $2(11.6-18.2)$ & Tertile 3 (>18.2) & \\
\hline No. of participants & 783 & 782 & 785 & \\
\hline Age (years) & $48.87 \pm 9.94$ & $50.36 \pm 10.59$ & $49.15 \pm 11.29$ & 0.01 \\
\hline BMI $\left(\mathrm{kg} / \mathrm{m}^{2}\right)$ & $28.46 \pm 3.78$ & $28.43 \pm 3.89$ & $28.46 \pm 3.79$ & 0.98 \\
\hline $\mathrm{NC}(\mathrm{cm})$ & $40.04 \pm 3.89$ & $40.38 \pm 3.93$ & $40.76 \pm 3.60$ & $<0.01$ \\
\hline $\mathrm{WC}(\mathrm{cm})$ & $100.66 \pm 10.06$ & $101.24 \pm 10.74$ & $101.11 \pm 10.30$ & 0.51 \\
\hline SBP (mmHg) & $138.52 \pm 18.66$ & $139.65 \pm 18.89$ & $142.19 \pm 21.28$ & $<0.01$ \\
\hline DBP (mmHg) & $91.10 \pm 13.93$ & $91.04 \pm 13.08$ & $93.63 \pm 15.30$ & $<0.01$ \\
\hline Gender $(n(\%))$ & & & & $<0.01$ \\
\hline Female & $293(37.42 \%)$ & $264(33.76 \%)$ & $179(22.80 \%)$ & \\
\hline Male & $490(62.58 \%)$ & $518(66.24 \%)$ & $606(77.20 \%)$ & \\
\hline Smoking status $(n(\%))$ & & & & $<0.01$ \\
\hline Never & $484(61.81 \%)$ & $463(59.21 \%)$ & $393(50.06 \%)$ & \\
\hline Former & $72(9.20 \%)$ & $80(10.23 \%)$ & $97(12.36 \%)$ & \\
\hline Current & $227(28.99 \%)$ & $239(30.56 \%)$ & $295(37.58 \%)$ & \\
\hline Drinking status $(n(\%))$ & & & & 0.10 \\
\hline Never & $528(67.43 \%)$ & $527(67.90 \%)$ & $502(63.95 \%)$ & \\
\hline Former & $39(4.98 \%)$ & $54(6.91 \%)$ & $63(8.03 \%)$ & \\
\hline Current & $216(27.59 \%)$ & $197(25.19 \%)$ & $220(28.03 \%)$ & \\
\hline \multicolumn{5}{|l|}{ History of disease } \\
\hline Arrhythmia (n (\%)) & $109(13.92 \%)$ & $178(22.76 \%)$ & $153(19.49 \%)$ & $<0.01$ \\
\hline Diabetes $(n(\%))$ & $151(19.28 \%)$ & $150(18.77 \%)$ & $115(14.65 \%)$ & 0.06 \\
\hline \multicolumn{5}{|l|}{ Laboratory examinations } \\
\hline $\mathrm{TC}(\mathrm{mmol} / \mathrm{L})$ & $4.61 \pm 1.31$ & $4.47 \pm 0.95$ & $4.59 \pm 1.34$ & 0.06 \\
\hline TG $(\mathrm{mmol} / \mathrm{L})$ & $1.77(1.28-2.47)$ & $1.73(1.22-2.48)$ & $1.76(1.25-2.54)$ & 0.25 \\
\hline HDL-c (mmol/L) & $1.11 \pm 0.29$ & $1.11 \pm 0.31$ & $1.09 \pm 0.28$ & 0.32 \\
\hline $\mathrm{LDL}-\mathrm{c}(\mathrm{mmol} / \mathrm{L})$ & $2.61 \pm 0.81$ & $2.72 \pm 0.80$ & $2.63 \pm 0.81$ & 0.02 \\
\hline hs-CRP (mg/L) & $2.21(0.90-4.07)$ & $2.01(0.96-4.19)$ & $2.12(0.99-3.83)$ & 0.85 \\
\hline FPG (mmol/L) & $5.30 \pm 1.35$ & $5.33 \pm 1.59$ & $5.21 \pm 1.48$ & 0.26 \\
\hline Hcy $(\mu \mathrm{mol} / \mathrm{L})$ & $7.70 \pm 3.32$ & $14.70 \pm 1.87$ & $29.22 \pm 10.15$ & $<0.01$ \\
\hline $\mathrm{eGFR}\left(\mathrm{mL} / \mathrm{min} / 1.73 \mathrm{~m}^{2}\right)$ & $99.26 \pm 21.08$ & $95.75 \pm 20.50$ & $93.39 \pm 22.11$ & $<0.01$ \\
\hline $\mathrm{Cr}(\mu \mathrm{mol} / \mathrm{L})$ & $73.60 \pm 24.55$ & $76.21 \pm 20.98$ & $81.62 \pm 25.22$ & $<0.01$ \\
\hline \multicolumn{5}{|l|}{ Polysomnography examinations } \\
\hline AHI (events/hour) & $17.40(10.00-33.80)$ & $19.40(10.40-34.95)$ & $18.50(10.50-32.80)$ & 0.52 \\
\hline AI (events/hour) & $2.50(0.40-9.80)$ & $3.20(0.50-12.28)$ & $3.10(0.60-11.30)$ & 0.79 \\
\hline HI (events/hour) & $12.40(7.20-21.40)$ & $12.85(7.30-21.17)$ & $12.10(7.20-19.20)$ & 0.10 \\
\hline Sleep duration (minutes) & $368.17 \pm 73.55$ & $366.11 \pm 73.24$ & $367.05 \pm 72.27$ & 0.86 \\
\hline Mean $\mathrm{SaO}_{2}(\%)$ & $90.83 \pm 9.62$ & $91.35 \pm 6.86$ & $91.16 \pm 8.01$ & 0.46 \\
\hline Lowest $\mathrm{SaO}_{2}(\%)$ & $76.54 \pm 12.32$ & $77.12 \pm 10.00$ & $77.51 \pm 11.04$ & 0.23 \\
\hline \multicolumn{5}{|l|}{ Medication use } \\
\hline Antidiabetic drugs $(n(\%))$ & $133(16.99 \%)$ & $79(10.10 \%)$ & $102(12.99 \%)$ & $<0.01$ \\
\hline Lipid-lowering drugs $(n(\%))$ & $417(53.26 \%)$ & $536(68.54 \%)$ & $605(77.07 \%)$ & $<0.01$ \\
\hline Antihypertensive drugs $(n(\%))$ & $713(91.06 \%)$ & $737(94.25 \%)$ & $766(97.58 \%)$ & $<0.01$ \\
\hline Antiplatelet drugs $(n(\%))$ & $541(69.09 \%)$ & $620(79.28 \%)$ & $508(64.71 \%)$ & $<0.01$ \\
\hline Regular CPAP treatment $(n(\%))$ & $22(3.07 \%)$ & $41(6.27 \%)$ & $34(2.04 \%)$ & $<0.01$ \\
\hline Follow-up duration (days) & $2627.00(2313.00-2995.00)$ & $2655.00(2287.50-2984.75)$ & $2563.00(2265.00-2973.00)$ & 0.26 \\
\hline Incident ischemic stroke $(n(\%))$ & & & & $<0.01$ \\
\hline
\end{tabular}


TABLE 1: Continued.

\begin{tabular}{cccc}
\hline Variable & & Hcy tertiles $(\mathrm{mmol} / \mathrm{L})$ & Tertile 3 $(>18.2)$ \\
\hline No & Tertile $1(<11.6)$ & $743(95.01 \%)$ & $748(95.29 \%)$ \\
Yes & $768(98.08 \%)$ & $39(4.99 \%)$ & $37(4.71 \%)$ \\
\hline
\end{tabular}

Data are $n(\%)$, mean \pm SD, or median (interquartile range). BMI: body mass index; NC: neck circumference; WC: waist circumference; SBP: systolic blood pressure; DBP: diastolic blood pressure; TC: total cholesterol; TG: triglyceride; HDL-c: high-density lipoprotein cholesterol; LDL-c: low-density lipoprotein cholesterol; hs-CRP: high-sensitivity C-reactive protein; FPG: fasting plasma glucose; Hcy: homocysteine; eGFR: estimated glomerular filtration rate; Cr: creatinine; AHI: apnea hypopnea index; $\mathrm{AI}$ : apnea index; $\mathrm{HI}$ : hypopnea index; mean $\mathrm{SaO}_{2}$ : mean oxygen saturation; lowest $\mathrm{SaO}_{2}$ : lowest oxygen saturation; CPAP: continuous positive airway pressure.

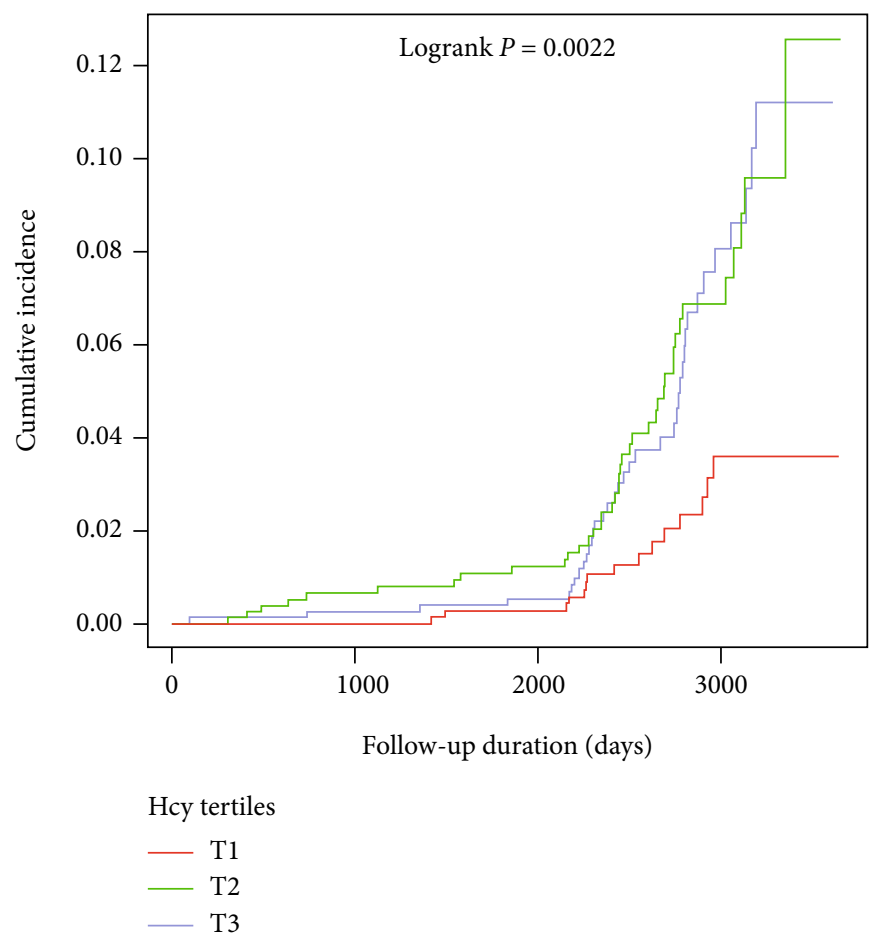

FIgURE 2: Kaplan-Meier curves of incidence of ischemic stroke according to tertiles of baseline plasma Hcy concentrations.

positively associated with the first occurrence of IS (Table 2). In the sensitivity analysis, all $E$-values were more than one, indicating that the current association tends to be stable and that our main findings are unlikely to be offset by unmeasured confounding variables. Moreover, when plasma Hcy concentrations were divided into different categories, there were obvious changes in effect sizes. The risk of the first IS increased over time by baseline plasma Hcy concentration tertiles and was still significant despite adjustment for potential confounders, and the fully adjusted HRs (adjusted model III) were 2.13 (95\% CI: 1.74-2.59) and 1.76 (95\% CI: 1.43-2.17) for tertiles 2 and 3, respectively, versus tertile 1 of the baseline plasma Hcy concentrations.

\subsection{Threshold Effect Analysis of Plasma Hcy Concentrations} on Incident First IS. After adjusting for potential confounders, a nonlinear relationship was observed between plasma Hcy concentration and the first IS (Figure 3). The inflection point, determined by the two-piecewise recursive algorithm and linear regression, was $5 \mu \mathrm{mol} / \mathrm{L}$. The $P$ value of the log-likelihood ratio test was less than 0.01 , indicating that the two-piecewise linear regression was more appropriate for fitting the relationship between plasma Hcy concentrations and the risk of the first IS. On the right of the inflection point (plasma Hcy concentrations $\geq 5 \mu \mathrm{mol} / \mathrm{L}$ ), we observed a positive association between plasma Hcy concentrations and the occurrence of the first IS ( $\mathrm{HR}=1.35$, 95\% CI: 1.08-1.69, $P<0.01)$. On the left side of the inflection point (plasma Hcy concentrations $<5 \mu \mathrm{mol} / \mathrm{L}$ ), however, their relationship saturated $(\mathrm{HR}=1.02,95 \% \mathrm{CI}: 0.97-1.07$, $P=0.28$ ) (Table 3).

3.5. Stratified Analyses. To better identify plasma Hcy concentrations and other possible influences on the risk of the first IS, we performed stratified analyses and interaction tests in prespecified subgroups. In stratified analyses, a greater positive correlation between baseline plasma Hcy concentrations and new-onset IS was observed for participants with $\mathrm{DBP} \geq 90 \mathrm{mmHg}$ (per SD increment; $\mathrm{HR}=1.48,95 \% \mathrm{CI}$ : 1.33-1.65 vs. <90 mmHg: $\mathrm{HR}=1.20,95 \% \mathrm{CI}: 1.02-1.42, P$ - 
TABLE 2: Association between Hcy and incident of the first IS in different models.

\begin{tabular}{|c|c|c|c|c|}
\hline Exposure & $\begin{array}{l}\text { Crude model (HR to } 95 \% \\
\text { CI to } P \text { ) }\end{array}$ & $\begin{array}{l}\text { Adjust model I (HR to } 95 \% \\
\text { CI to } P \text { ) }\end{array}$ & $\begin{array}{l}\text { Adjust model II (HR to } 95 \% \\
\text { CI to } P \text { ) }\end{array}$ & $\begin{array}{l}\text { Adjust model III (HR to } 95 \% \\
\text { CI to } P \text { ) }\end{array}$ \\
\hline \multicolumn{5}{|l|}{ Continuous } \\
\hline $\begin{array}{l}\text { Hcy (per SD } \\
\text { increment) }\end{array}$ & $1.37(1.30$ to 1.44$)<0.01$ & $1.35(1.27$ to 1.42$)<0.01$ & $1.34(1.26$ to 1.43$)<0.01$ & $1.32(1.24$ to 1.38$)<0.01$ \\
\hline \multicolumn{5}{|l|}{ Categorical } \\
\hline \multicolumn{5}{|l|}{$\begin{array}{l}\text { Hcy tertiles } \\
(\mu \mathrm{mol} / \mathrm{L})\end{array}$} \\
\hline $\begin{array}{l}\text { Tertile } 1 \\
(<11.6)\end{array}$ & Reference & Reference & Reference & Reference \\
\hline $\begin{array}{r}\text { Tertile } 2 \\
(11.6-18.2)\end{array}$ & $2.91(2.42$ to 3.49$)<0.01$ & $2.67(2.22$ to 3.20$)<0.01$ & $2.33(1.94$ to 2.72$)<0.01$ & $2.13(1.74$ to 2.59$)<0.01$ \\
\hline $\begin{array}{l}\text { Tertile } 3 \\
(>18.2)\end{array}$ & $2.75(2.29$ to 3.31$)<0.01$ & $2.23(1.85$ to 2.70$)<0.01$ & $1.96(1.73$ to 2.19$)<0.01$ & $1.76(1.43$ to 2.17$)<0.01$ \\
\hline
\end{tabular}

Crude model: adjusted for none. Adjust model I: adjusted for age and gender at baseline. Adjust model II: adjusted for variables in adjusted model I plus smoking status, drinking status, NC, WC, and BMI at baseline. Adjust model III: fully adjusted model. Adjusted for variables in adjusted model II plus SBP, DBP, history of arrhythmia and diabetes, AHI, AI, HI, sleep duration, mean $\mathrm{SaO}_{2}$ and lowest $\mathrm{SaO}_{2}$, antidiabetic drugs, antiplatelet drugs, lipidlowering drugs, regular CPAP treatment, antihypertensive drugs, and TC, TG, HDL-c, LDL-c, FPG, eGFR, Cr, and hs-CRP levels at baseline. Abbreviations are the same as in Table 1.

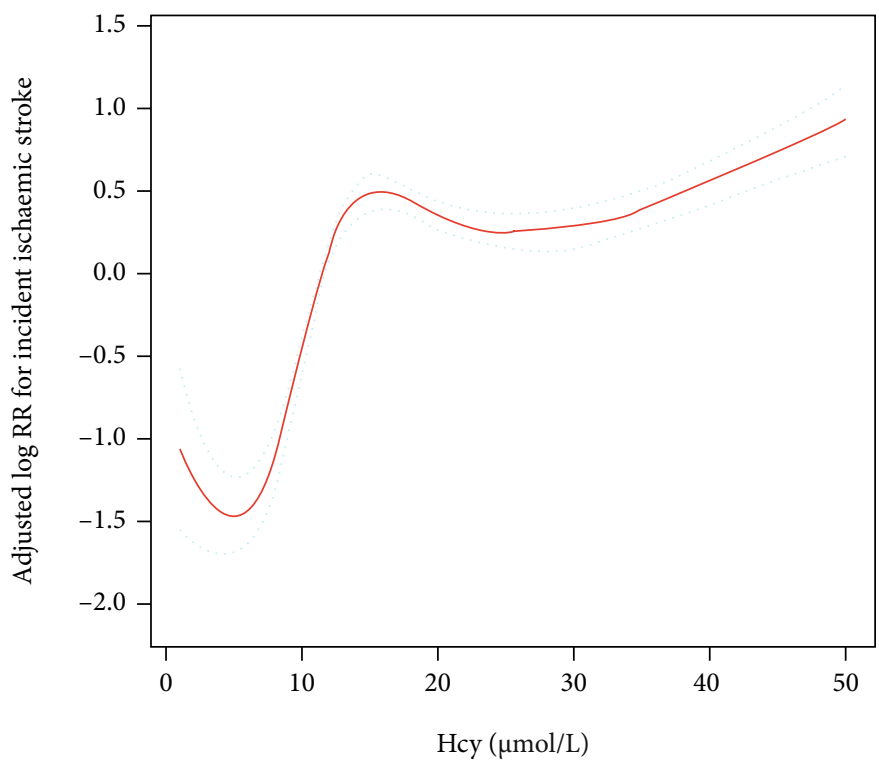

FIGURE 3: A piecewise linear regression model was used to detect the association of plasma Hcy concentrations and the first IS according to the plasma Hcy concentration cut points. All were adjusted for age, gender, smoking status, drinking status, NC, WC, BMI, SBP, DBP, history of arrhythmia, diabetes, AHI, AI, HI, sleep duration, mean $\mathrm{SaO}_{2}$ and lowest $\mathrm{SaO}_{2}$, antidiabetic drugs, antiplatelet drugs, lipidlowering drugs, regular CPAP treatment, antihypertensive drugs, and TC, TG, HDL-c, LDL-c, FPG, eGFR, Cr, and hs-CRP levels at baseline.

interaction $=0.04$ ) and $\mathrm{BMI} \geq 24$ and $<28 \mathrm{~kg} / \mathrm{m}^{2}$ (per SD increment; $\mathrm{HR}=1.46,95 \% \mathrm{CI}: 1.26-1.70$ vs. $<24 \mathrm{~kg} / \mathrm{m}^{2}: \mathrm{HR}$ $=1.13,95 \%$ CI: $0.95-1.33$ vs. $\geq 28 \mathrm{~kg} / \mathrm{m}^{2}: \mathrm{HR}=1.46,95 \%$ CI: $1.25-1.70, P$-interaction $=0.03) \quad($ Table 4$)$. However, other variables, including gender (female vs. male), diabetes (no vs. yes), smoking status (never vs. ever vs. current), arrhythmia (no vs. yes), drinking status (never vs. ever vs. current), age $(<60$ vs. $\geq 60$ years), FPG $(<6.1$ vs. $\geq 6.1 \mathrm{mmol} / \mathrm{L}$ ), SBP (<140 vs. $\geq 140 \mathrm{mmHg}$ ), AHI ( $\geq 5$ and $<15$ vs. $\geq 15$ and $<30$ vs. $\geq 30$ events/hour), and eGFR ( $<90$ vs. $\geq 90 \mathrm{~mL} / \mathrm{min} / 1.73 \mathrm{~m}^{2}$ ) at baseline, did not dramatically modify the relationship between plasma Hcy concentrations and the first IS (all $P$-interactions $>0.05$ ) (Table 4 ).

\section{Discussion}

In China, IS is the most prevalent type of cerebrovascular event. Therefore, the prevention of IS is an important and urgent public health issue $[6,7,32]$. Since the discovery of the association of Hcy with the pathogenesis of atherosclerosis, Hcy-lowering therapies have attracted considerable attention among the various prevention strategies for IS 
[33, 34]. In addition, a compelling and developing body of epidemiological evidence demonstrates a significant correlation between increased Hcy concentrations and enhanced risk of IS [35, 36]. However, the relationship between Hcy and IS is inconclusive. Iso et al. conducted a prospective, nested case-control study of 11846 Japanese subjects aged 40 to 85 years. Their findings showed odds ratios $(95 \% \mathrm{CI})$ of $3.89(1.60$ to 9.46$)$ for the highest $(\geq 11.0 \mu \mathrm{mol} / \mathrm{L})$ versus the lowest quartile $(<7.0 \mu \mathrm{mol} / \mathrm{L})$ of Hcy for IS after adjusting for cardiovascular risk factors. The respective odds ratio associated with a $5 \mu \mathrm{mol} / \mathrm{L}$ increase in Hcy was 1.52 (1.07 to 2.14) [37]. Sacco et al. followed a population-based cohort for vascular events. Their findings suggest that Hcy elevations above $15 \mu \mathrm{mol} / \mathrm{L}$ are an independent risk factor for IS, whereas mild tHcy elevations of 10 to $15 \mu \mathrm{mol} / \mathrm{L}$ are not predictive. Hcy has the greatest vascular impact in Whites and Hispanics and less in Blacks [38]. However, the Caerphilly study conducted by Fallon et al. yielded the opposite results to the above study. A total of 2254 male participants aged 50 to 64 years were included in the Caerphilly study, and after a mean follow-up of 10.2 years, a total of 107 participants experienced IS. However, after adjusting for confounding, no significant association was found between Hcy and IS [39].

In the current study, we found a meaningful relationship between plasma Hcy concentrations and the incidence of the first IS in hypertensive patients with OSA. Similar results have been reported in several previous studies in patients with simple hypertension or healthy populations, but these studies did not identify a nonlinear association [38, 40,41]. We found a threshold saturation effect association between plasma Hcy concentrations and the first IS even after removing adjusted covariates from the model. This is the first study to explore the nonlinear association between plasma Hcy concentration and the first IS, calculated for plasma Hcy concentrations at the inflection points of 5 and $15 \mu \mathrm{mol} / \mathrm{L}$. Notably, this association between plasma Hcy concentrations and the first IS has opposite effects in the middle of the two inflection points versus the left and right sides. Plasma Hcy concentrations were positively associated with the risk of the first IS when plasma Hcy concentrations were between 5 and $15 \mu \mathrm{mol} / \mathrm{L}$. This suggests that the risk of the first IS in hypertensive patients with OSA increases rapidly when plasma Hcy concentrations are between 5 and $15 \mu \mathrm{mol} / \mathrm{L}$. In contrast, there was no significant association between plasma Hcy concentrations and the risk of the first IS when plasma Hcy concentrations were $<5 \mu \mathrm{mol} / \mathrm{L}$ or $>15 \mu \mathrm{mol} / \mathrm{L}$. However, the mechanism behind the threshold saturation effect association between plasma Hcy concentrations and the first IS and the inflection point is not clear. To better understand the association between plasma Hcy concentrations and the risk of the first IS, we included significant variables in univariate analysis and noncollinear variables in the multivariate analysis. After adjustment for covariates, participants in the highest tertile of plasma Hcy concentrations had a 1.76 -fold greater risk of the first IS compared with those in the lowest tertile.

Subgroup analysis and exploration of interactions are essential for clinical research to better understand plasma
TABLE 3: A piecewise linear regression model was applied to detect the association of Hcy and IS according to the Hcy cut points.

\begin{tabular}{lcc}
\hline Outcome: incident of ischemic stroke & HR $(95 \% \mathrm{CI})$ & $\begin{array}{c}P \\
\text { value }\end{array}$ \\
\hline Linear regression & $1.13(1.02$ to & $<0.01$ \\
Two-piecewise linear regression & $1.24)$ & \\
model & & \\
Hcy $<5 \mu \mathrm{mol} / \mathrm{L}$ & $1.02(0.97$ to & 0.28 \\
& $1.07)$ & \\
Hcy $\geq 5 \mu \mathrm{mol} / \mathrm{L}$ & $1.35(1.08$ to & $<0.01$ \\
$P$ for the $\log$-likelihood ratio test & $1.69)$ & \\
\hline
\end{tabular}

Notes: adjusted for age, gender, smoking status, drinking status, NC, WC, BMI, SBP, DBP, history of arrhythmia, diabetes, AHI, AI, HI, sleep duration, mean $\mathrm{SaO} 2$ and lowest $\mathrm{SaO} 2$, antidiabetic drugs, antiplatelet drugs, lipid-lowering drugs, regular CPAP treatment, antihypertensive drugs, and TC, TG, HDL-c, LDL-c, FPG, eGFR, Cr, and hs-CRP levels at baseline. Abbreviations are the same as in Table 1.

Hcy concentrations and the risk of the first IS in different populations. In this study, these factors, including gender, diabetes, coronary heart disease, arrhythmia, smoking status, drinking status, age, BMI, FPG, SBP, DBP, AHI, and eGFR, and stronger correlations were identified in participants with $\mathrm{BMI} \geq 24 \mathrm{~kg} / \mathrm{m}^{2}$ and DBP $\geq 90 \mathrm{mmHg}$. In China, the prevalence of obesity in hypertensive patients with OSA is high [42]. A meta-analysis identified a $40 \%$ increase in IS mortality for every $5 \mathrm{~kg} / \mathrm{m}^{2}$ increase in BMI among those with a BMI between 25 and $50 \mathrm{~kg} / \mathrm{m}^{2}$. However, there was no association between BMI and IS mortality in those with a BMI of $15-24 \mathrm{~kg} / \mathrm{m}^{2}$ [43]. The exact biological mechanism of the interaction between high BMI and high Hcy is unclear. In available studies, a reasonable biological interpretation of this interaction may be due to the fact that elevated Hcy and obesity may share several cellular and molecular mechanisms (e.g., impaired mitochondrial function and the onset of oxidative stress) that are responsible for the development of stroke $[44,45]$. In addition, we found that baseline DBP $(\geq 90 \mathrm{mmHg}$ vs. $<90 \mathrm{mmHg}$ ) significantly altered the effect of Hcy on the risk of the first IS. Zheng et al. [46] found that in Chinese patients with uncontrolled hypertension, the prevalence of IS increased with increasing DBP, suggesting that DBP has an important contribution to the incidence of IS in hypertensive patients. This suggests that DBP serves an essential role in the incidence of IS in hypertensive patients. DBP is traditionally thought to reflect structural changes in small arteries or thinning of microvessels. Thus, elevated DBP may reflect dysfunction of peripheral microvessels, whereas elevated Hcy reflects damage to large- and medium-sized arteries; thus, elevated levels of Hcy and DBP may reflect damage to peripheral vessels and largeand medium-sized arteries, which helps explain the apparent concerted impact of DBP and Hcy on the risk of the first IS $[47,48]$. The optimal blood pressure level for controlling Hcy on the risk of the first IS should be explored in future studies. 
TABLE 4: Association between Hcy and the first IS in various subgroups.

\begin{tabular}{|c|c|c|c|c|c|c|}
\hline Stratification variable & No. of participants. & HR (95\% CI) & $95 \%$ CI low & 95\% CI high & $P$ value & $P$ for interaction \\
\hline Gender & & & & & & 0.63 \\
\hline Female & 744 & 1.20 & 0.65 & 2.22 & 0.56 & \\
\hline Male & 1606 & 1.40 & 1.13 & 1.74 & $<0.01$ & \\
\hline Diabetes & & & & & & 0.51 \\
\hline No & 1937 & 1.39 & 1.12 & 1.73 & $<0.01$ & \\
\hline Yes & 413 & 1.16 & 0.70 & 1.92 & 0.57 & \\
\hline Arrhythmia & & & & & & 0.18 \\
\hline No & 2306 & 1.24 & 1.05 & 1.46 & 0.01 & \\
\hline Yes & 440 & 1.41 & 1.27 & 1.57 & $<0.01$ & \\
\hline Smoking status & & & & & & 0.63 \\
\hline Never & 1342 & 1.33 & 0.94 & 1.88 & 0.10 & \\
\hline Former & 258 & 1.78 & 1.04 & 3.05 & 0.04 & \\
\hline Current & 750 & 1.34 & 0.99 & 1.80 & 0.05 & \\
\hline Drinking status & & & & & & 0.65 \\
\hline Never & 1557 & 1.43 & 1.12 & 1.82 & $<0.01$ & \\
\hline Former & 166 & 1.24 & 0.63 & 2.46 & 0.54 & \\
\hline Current & 627 & 1.13 & 0.72 & 1.79 & 0.59 & \\
\hline Age (years) & & & & & & 0.87 \\
\hline$<60$ & 1911 & 1.36 & 1.07 & 1.73 & 0.01 & \\
\hline$\geq 60$ & 439 & 1.42 & 0.96 & 2.08 & 0.08 & \\
\hline BMI $\left(\mathrm{kg} / \mathrm{m}^{2}\right)$ & & & & & & 0.03 \\
\hline$<24$ & 221 & 1.13 & 0.95 & 1.33 & 0.16 & \\
\hline$\geq 24,<28$ & 934 & 1.46 & 1.26 & 1.70 & $<0.01$ & \\
\hline$\geq 28$ & 1195 & 1.46 & 1.25 & 1.70 & $<0.01$ & \\
\hline $\mathrm{SBP}(\mathrm{mmHg})$ & & & & & & 0.41 \\
\hline$<140$ & 1084 & 1.25 & 0.86 & 1.82 & 0.23 & \\
\hline$\geq 140$ & 1266 & 1.51 & 1.18 & 1.92 & $<0.01$ & \\
\hline $\mathrm{DBP}(\mathrm{mmHg})$ & & & & & & 0.04 \\
\hline$<90$ & 887 & 1.20 & 1.02 & 1.42 & 0.03 & \\
\hline$\geq 90$ & 1463 & 1.48 & 1.33 & 1.65 & $<0.01$ & \\
\hline AHI (events/hour) & & & & & & 0.37 \\
\hline$\geq 5,<15$ & 934 & 1.46 & 1.04 & 2.07 & 0.03 & \\
\hline$\geq 15,<30$ & 722 & 1.47 & 1.04 & 2.09 & 0.03 & \\
\hline$\geq 30$ & 694 & 1.07 & 0.73 & 1.56 & 0.74 & \\
\hline $\mathrm{FPG}(\mathrm{mmol} / \mathrm{L})$ & & & & & & 0.72 \\
\hline$<6.1$ & 1991 & 1.38 & 1.23 & 1.54 & $<0.01$ & \\
\hline$\geq 6.1$ & 359 & 1.32 & 1.04 & 1.66 & 0.02 & \\
\hline $\mathrm{eGFR}\left(\mathrm{mL} / \mathrm{min} / 1.73 \mathrm{~m}^{2}\right)$ & & & & & & 0.59 \\
\hline$<90$ & 941 & 1.42 & 1.08 & 1.85 & 0.01 & \\
\hline$\geq 90$ & 1409 & 1.27 & 0.93 & 1.72 & 0.13 & \\
\hline
\end{tabular}

Note 1: adjusted for age, gender, smoking status, drinking status, NC, WC, BMI, SBP, DBP, history of arrhythmia, diabetes, AHI, AI, HI, sleep duration, mean $\mathrm{SaO}_{2}$ and lowest $\mathrm{SaO}_{2}$, antidiabetic drugs, antiplatelet drugs, lipid-lowering drugs, regular CPAP treatment, antihypertensive drugs, and TC, TG, HDL-c, LDLc, FPG, eGFR, Cr, and hs-CRP levels at baseline. Note 2: in each case, the model was not adjusted for hierarchical covariates. Abbreviations are the same as in Table 1.

Our findings may have important implications for public health concerns $[19,49]$. In hypertensive patients with OSA, increased Hcy levels are probably a changeable risk factor for IS. Lowering Hcy levels may reduce the risk of stroke in hypertensive patients with OSA, and the prog- nostic effect of Hcy levels has important clinical implications. However, the relationship between slightly increased Hcy levels and the risk of IS is disputable [38]. Our study revealed a nonlinear dose-response relationship between Hcy levels and the first IS in hypertensive patients 
with OSA, and slightly increased Hcy levels may increase the risk of the first IS in hypertensive patients with OSA. However, a few investigations have shown that slightly increased Hcy levels do not enhance the risk of IS [38, 50]. Further, in IS patients with marginal or slightly increased Hcy levels, reducing Hcy with high-dose vitamin therapy did not reduce the risk of IS recurrence [51]. Therefore, additional investigations are necessary to verify whether the risk of IS in hypertensive patients with OSA can be reduced by intensive control or maintenance of lower Hcy levels through folic acid and vitamin B12 supplementation.

The present study has some unique strengths. First, it is the first report of a nonlinear relationship between Hcy and the first IS in hypertensive patients with OSA. Second, the present study is a retrospective cohort research and thus vulnerable to potential influences. However, we applied rigorous statistical adjustments to reduce remnant confounders as much as possible. Finally, we conducted subgroup analyses and interaction tests to further demonstrate reliability for the results and to identify potential interactions with other variables.

Limitations of the study are mainly in the areas of the following. First, levels of Hcy were tested only once at baseline, so we were unable to investigate the effect of changes in levels of Hcy on IS. Second, levels of Hcy could be affected by genetic background, dietary habits, and/or medication usage. It is possible that a single plasma Hcy concentration test may not provide us with sufficient information to determine a causal relationship between Hcy and IS. Third, participants were limited to hypertensive patients with OSA in northwest China. Therefore, the findings of the present investigation should be approached with caution for extrapolation to the general population or other ethnic communities. Fourth, data on adiponectin, IL-6, and TNF- $\alpha$ levels are lacking in this cohort study, so it is not possible to compare the accuracy of Hcy levels and other biomarkers for predicting the risk of IS $[2,5]$. Finally, recall bias may have existed during data collection. However, recall bias was minimized during data collection through rigorous training in survey methodology and the application of standard operating procedures.

\section{Conclusion}

In conclusion, elevated plasma Hcy concentrations were independently associated with the risk of the first IS in hypertensive patients with OSA. Plasma Hcy concentrations $\geq 5 \mu \mathrm{mol} / \mathrm{L}$ obviously increase the risk of the first IS in hypertensive patients with OSA. Undoubtedly, lowering plasma Hcy concentrations to $<5 \mu \mathrm{mol} / \mathrm{L}$ is an efficient and modest approach to minimize the risk of the first IS in hypertensive patients with OSA.

\section{Data Availability}

All relative data are in the paper.

\section{Ethical Approval}

This study was approved by the Ethics Committee of the People's Hospital of Xinjiang Uygur Autonomous Region (no. 2019030662).

\section{Conflicts of Interest}

The authors declare that they have no conflicts of interest.

\section{Authors' Contributions}

Nanfang Li and Xintian Cai contributed equally to this paper.

\section{Acknowledgments}

This work was financially supported by the Special Research Project for Young Medical Science and Technology Talents (No. WJWY-202031) and the Research Project of Xinjiang People's Hospital (No. 20190419).

\section{References}

[1] J. D. Pandian, S. L. Gall, M. P. Kate et al., "Prevention of stroke: a global perspective," Lancet, vol. 392, no. 10154, pp. 12691278, 2018.

[2] W. J. Tu, H. C. Qiu, Y. K. Liu, Q. Liu, X. Zeng, and J. Zhao, "Elevated levels of adiponectin associated with major adverse cardiovascular and cerebrovascular events and mortality risk in ischemic stroke," Cardiovascular Diabetology, vol. 19, no. 1, p. $125,2020$.

[3] W. J. Tu, B. H. Chao, L. Ma et al., "Case-fatality, disability and recurrence rates after first-ever stroke: A study from bigdata observatory platform for stroke of China," Brain Research Bulletin, vol. 175, pp. 130-135, 2021.

[4] S. Wu, B. Wu, M. Liu et al., "Stroke in China: advances and challenges in epidemiology, prevention, and management," The Lancet Neurology, vol. 18, no. 4, pp. 394-405, 2019.

[5] L. S. Cheng, W. J. Tu, Y. Shen, L. J. Zhang, and K. Ji, "Combination of high-sensitivity C-reactive protein and homocysteine predicts the post-stroke depression in patients with ischemic stroke," Molecular Neurobiology, vol. 55, no. 4, pp. 29522958, 2018.

[6] T. Li, J. Zhu, Q. Fang et al., "Association of H-type hypertension with stroke severity and prognosis," BioMed Research International, vol. 2018, Article ID 8725908, 7 pages, 2018.

[7] C. Wang, Z. du, N. Ye et al., "Using the atherogenic index of plasma to estimate the prevalence of ischemic stroke within a general population in a rural area of China," BioMed Research International, vol. 2020, Article ID 7197054, 7 pages, 2020.

[8] A. S. Jordan, D. G. McSharry, and A. Malhotra, "Adult obstructive sleep apnoea," Lancet, vol. 383, no. 9918, pp. 736-747, 2014.

[9] P. Lévy, M. Kohler, W. T. McNicholas et al., "Obstructive sleep apnoea syndrome," Nature Reviews. Disease Primers, vol. 1, no. 1, article 15015, 2015.

[10] H. Hou, Y. Zhao, W. Yu et al., "Association of obstructive sleep apnea with hypertension: a systematic review and meta-analysis," Journal of Global Health, vol. 8, no. 1, article 010405, 2018. 
[11] M. A. Martínez-García, C. Navarro-Soriano, G. Torres et al., "Beyond resistant hypertension," Hypertension, vol. 72, no. 3, pp. 618-624, 2018.

[12] R. P. Pedrosa, L. F. Drager, C. C. Gonzaga et al., "Obstructive sleep apnea: the most common secondary cause of hypertension associated with resistant hypertension," Hypertension, vol. 58, no. 5, pp. 811-817, 2011.

[13] L. A. Salman, R. Shulman, and J. B. Cohen, "Obstructive sleep apnea, hypertension, and cardiovascular risk: epidemiology, pathophysiology, and management," Current Cardiology Reports, vol. 22, no. 2, p. 6, 2020.

[14] I. Cano-Pumarega, J. Durán-Cantolla, F. Aizpuru et al., "Obstructive sleep apnea and systemic hypertension: longitudinal study in the general population: the Vitoria Sleep Cohort," American Journal of Respiratory and Critical Care Medicine, vol. 184, no. 11, pp. 1299-1304, 2011.

[15] E. Zhao, S. Chen, Y. Du, and Y. Zhang, "Association between sleep apnea hypopnea syndrome and the risk of atrial fibrillation: a meta-analysis of cohort study," BioMed Research International, vol. 2018, Article ID 5215868, 2018.

[16] J. Y. Dong, Y. H. Zhang, and L. Q. Qin, “Obstructive sleep apnea and cardiovascular risk: meta-analysis of prospective cohort studies," Atherosclerosis, vol. 229, no. 2, pp. 489-495, 2013.

[17] Y. K. Loke, J. W. Brown, C. S. Kwok, A. Niruban, and P. K. Myint, "Association of obstructive sleep apnea with risk of serious cardiovascular events: a systematic review and metaanalysis," Circulation. Cardiovascular Quality and Outcomes, vol. 5, no. 5, pp. 720-728, 2012.

[18] C. Xie, R. Zhu, Y. Tian, and K. Wang, "Association of obstructive sleep apnoea with the risk of vascular outcomes and allcause mortality: a meta-analysis," BMJ open, vol. 7, no. 12, article e013983, 2017.

[19] X. Wu, Q. Zhou, Q. Chen et al., "Association of homocysteine level with risk of stroke: A dose-response meta- analysis of prospective cohort studies," Nutrition, Metabolism, and Cardiovascular Diseases, vol. 30, no. 11, pp. 1861-1869, 2020.

[20] S. S. Kang and R. S. Rosenson, "Analytic approaches for the treatment of hyperhomocysteinemia and its impact on vascular disease," Cardiovascular Drugs and Therapy, vol. 32, no. 2, pp. 233-240, 2018.

[21] B. Yang, S. Fan, X. Zhi et al., "Prevalence of hyperhomocysteinemia in China: a systematic review and meta-analysis," Nutrients, vol. 7, no. 1, pp. 74-90, 2015.

[22] J. Skeete and D. J. DiPette, "Relationship between homocysteine and hypertension: new data add to the debate," The Journal of Clinical Hypertension, vol. 19, no. 11, pp. 1171-1172, 2017.

[23] R. L. Sacco, R. Adams, G. Albers et al., "Guidelines for prevention of stroke in patients with ischemic stroke or transient ischemic attack: a statement for healthcare professionals from the American Heart Association/American Stroke Association Council on Stroke: co-sponsored by the Council on Cardiovascular Radiology and Intervention: the American Academy of Neurology affirms the value of this guideline," Circulation, vol. 113, no. 10, pp. e409-e449, 2006.

[24] W. J. Powers, A. A. Rabinstein, T. Ackerson et al., "Guidelines for the early management of patients with acute ischemic stroke: 2019 update to the 2018 guidelines for the early management of acute ischemic stroke: a guideline for healthcare professionals from the American Heart Association/American Stroke Association," Stroke, vol. 50, no. 12, pp. e344-e418, 2019.
[25] W. Yang, L. Shao, M. Heizhati et al., "Oropharyngeal microbiome in obstructive sleep apnea: decreased diversity and abundance," Journal of Clinical Sleep Medicine, vol. 15, no. 12, pp. 1777-1788, 2019.

[26] L. Wang, N. Li, X. Yao et al., "Detection of secondary causes and coexisting diseases in hypertensive patients: OSA and PA are the common causes associated with hypertension," BioMed Research International, vol. 2017, Article ID 8295010, 8 pages, 2017.

[27] A. S. Levey, L. A. Inker, and J. Coresh, "GFR estimation: from physiology to public health," American Journal of Kidney Diseases, vol. 63, no. 5, pp. 820-834, 2014.

[28] Y. Yu, L. Liu, J. Huang et al., "Association between systolic blood pressure and first ischemic stroke in the Chinese older hypertensive population," The Journal of International Medical Research, vol. 48, no. 4, p. $030006052092009,2020$.

[29] Y. Q. Huang, K. Lo, X. C. Liu, S. T. Tang, C. Huang, and Y. Q. Feng, "The relationship between fasting blood glucose levels and first ischemic stroke in elderly hypertensive patients," Risk Management and Healthcare Policy, vol. Volume 13, pp. 777784, 2020.

[30] E. von Elm, D. G. Altman, M. Egger, S. J. Pocock, P. C. Gøtzsche, and J. P. Vandenbroucke, "The strengthening the reporting of observational studies in epidemiology (STROBE) statement: guidelines for reporting observational studies," Lancet, vol. 370, no. 9596, pp. 1453-1457, 2007.

[31] J. Wang, D. Zhou, and X. Li, "The association between neutrophil-to-lymphocyte ratio and diabetic depression in U.S. adults with diabetes: findings from the 2009-2016 National Health and Nutrition Examination Survey (NHANES)," BioMed Research International, vol. 2020, Article ID 8297628, 7 pages, 2020.

[32] T. Li, X. Liu, S. Diao et al., "H-type hypertension is a risk factor for cerebral small-vessel disease," BioMed Research International, vol. 2020, Article ID 6498903, 6 pages, 2020.

[33] D. S. Wald, M. Law, and J. K. Morris, "Homocysteine and cardiovascular disease: evidence on causality from a meta-analysis," BMJ, vol. 325, no. 7374, pp. 1202-1206, 2002.

[34] G. N. Welch and J. Loscalzo, "Homocysteine and atherothrombosis," The New England Journal of Medicine, vol. 338, no. 15, pp. 1042-1050, 1998.

[35] Y. Huo, J. Li, X. Qin et al., "Efficacy of folic acid therapy in primary prevention of stroke among adults with hypertension in China: the CSPPT randomized clinical trial," JAMA, vol. 313, no. 13, pp. 1325-1335, 2015.

[36] T. Zhang, T. Lin, Y. Wang et al., "Estimated stroke-free survival of folic acid therapy for hypertensive adults: projection based on the CSPPT," Hypertension, vol. 75, no. 2, pp. 339346, 2020.

[37] H. Iso, Y. Moriyama, S. Sato et al., "Serum total homocysteine concentrations and risk of stroke and its subtypes in Japanese," Circulation, vol. 109, no. 22, pp. 2766-2772, 2004.

[38] R. L. Sacco, K. Anand, H. S. Lee et al., "Homocysteine and the risk of ischemic stroke in a triethnic cohort: the NOrthern MAnhattan Study," Stroke, vol. 35, no. 10, pp. 2263-2269, 2004.

[39] U. B. Fallon, P. Elwood, Y. Ben-Shlomo, J. B. Ubbink, R. Greenwood, and G. D. Smith, "Homocysteine and ischaemic stroke in men: the Caerphilly study," Journal of Epidemiology and Community Health, vol. 55, no. 2, pp. 91-96, 2001. 
[40] R. Kawamoto, T. Kajiwara, Y. Oka, and Y. Takagi, “An association between plasma homocysteine concentrations and ischemic stroke in elderly Japanese," Journal of Atherosclerosis and Thrombosis, vol. 9, no. 2, pp. 121-125, 2002.

[41] L. Han, Q. Wu, C. Wang et al., "Homocysteine, ischemic stroke, and coronary heart disease in hypertensive patients: a population-based, prospective cohort study," Stroke, vol. 46, no. 7, pp. 1777-1786, 2015.

[42] X. Qin, Y. Zhang, Y. Cai et al., "Prevalence of obesity, abdominal obesity and associated factors in hypertensive adults aged 45-75 years," Clinical Nutrition, vol. 32, no. 3, pp. 361-367, 2013.

[43] Prospective Studies Collaboration, G. Whitlock, S. Lewington et al., "Body-mass index and cause-specific mortality in 900 000 adults: collaborative analyses of 57 prospective studies," Lancet, vol. 373, no. 9669, pp. 1083-1096, 2009.

[44] A. Laha, A. Majumder, M. Singh, and S. C. Tyagi, "Connecting homocysteine and obesity through pyroptosis, gut microbiome, epigenetics, peroxisome proliferator-activated receptor $\gamma$, and zinc finger protein 407," Canadian Journal of Physiology and Pharmacology, vol. 96, no. 10, pp. 971-976, 2018.

[45] N. Komorniak, M. Szczuko, B. Kowalewski, and E. Stachowska, "Nutritional deficiencies, bariatric surgery, and serum homocysteine level: review of current literature," Obesity Surgery, vol. 29, no. 11, pp. 3735-3742, 2019.

[46] L. Zheng, Z. Sun, J. Li et al., "Pulse pressure and mean arterial pressure in relation to ischemic stroke among patients with uncontrolled hypertension in rural areas of China," Stroke, vol. 39, no. 7, pp. 1932-1937, 2008.

[47] M. J. Cipolla, D. S. Liebeskind, and S. L. Chan, "The importance of comorbidities in ischemic stroke: impact of hypertension on the cerebral circulation," Journal of Cerebral Blood Flow and Metabolism, vol. 38, no. 12, pp. 2129-2149, 2018.

[48] P. Sun, L. Liu, C. Liu et al., "Carotid intima-media thickness and the risk of first stroke in patients with hypertension," Stroke, vol. 51, no. 2, pp. 379-386, 2020.

[49] F. Pan, M. Heizhati, L. Wang et al., "Distribution characteristics of circulating homocysteine and folate and related factors in agriculture, stock-raising and urban populations: a crosssectional survey," Public Health Nutrition, vol. 24, no. 5, pp. 1001-1008, 2021.

[50] A. G. Bostom, I. H. Rosenberg, H. Silbershatz et al., "Nonfasting plasma total homocysteine levels and stroke incidence in elderly persons: the Framingham Study," Annals of Internal Medicine, vol. 131, no. 5, pp. 352-355, 1999.

[51] J. F. Toole, M. R. Malinow, L. E. Chambless et al., "Lowering homocysteine in patients with ischemic stroke to prevent recurrent stroke, myocardial infarction, and death: the vitamin intervention for stroke prevention (VISP) randomized controlled trial," JAMA, vol. 291, no. 5, pp. 565-575, 2004. 